\title{
BOUNDARY ANGULAR DERIVATIVES OF GENERALIZED SCHUR FUNCTIONS
}

\author{
VLADIMIR BOLOTNIKOV ${ }^{凶}$, TENGYAO WANG and JOSHUA M. WEISS
}

(Received 9 September 2011; accepted 17 October 2012; first published online 8 March 2013)

Communicated by P. C. Fenton

\begin{abstract}
Characterization of generalized Schur functions in terms of their Taylor coefficients was established by Krein and Langer ['Über einige Fortsetzungsprobleme, die eng mit der Theorie hermitescher Operatoren im Raume $\Pi_{\kappa}$ zusammenhängen. I. Einige Funktionenklassen und ihre Darstellungen', Math. Nachr. 77 (1977), 187-236]. We establish a boundary analogue of this characterization.
\end{abstract}

2010 Mathematics subject classification: primary 47A57; secondary 47A20, 47A48.

Keywords and phrases: boundary interpolation, angular derivatives, generalized Schur functions.

\section{Introduction}

Generalized Schur functions are functions of the form

$$
f(z)=\frac{s(z)}{b(z)},
$$

where the numerator $s$ is an analytic function mapping the open unit disk $\mathbb{D}$ into the closed unit disk (that is, $s$ is a Schur-class function) and where the denominator $b$ is a finite Blaschke product. Such functions appeared first in [15] in the interpolation context and were studied later in $[12,13]$. In what follows, we will write $\mathcal{S}, \mathcal{G} \mathcal{S}$ and $\mathcal{F B}$ for the set of Schur functions, the set of generalized Schur functions and the set of finite Blaschke products, respectively. Formula (1.1) is called the KreinLanger representation of a generalized Schur function $f$ (see [12]); the entries $s$ and $b$ are defined by $f$ uniquely up to a unimodular constant provided they have no common zeroes. Via nontangential boundary limits, the $\mathcal{G S}$-functions can be identified with the functions from the closed unit ball of $L^{\infty}(\mathbb{T})$ which admit meromorphic continuation inside the unit disk with a finite total pole multiplicity. The class $\mathcal{G} \mathcal{S}$

The research was partially supported by National Science Foundation Grant DMS 0901124.

(C) 2013 Australian Mathematical Publishing Association Inc. 1446-7887/2013 \$16.00 
can be alternatively defined as the class of functions $f$ meromorphic on $\mathbb{D}$ and such that the associated kernel

$$
K_{f}(z, \zeta):=\frac{1-f(z) \overline{f(\zeta)}}{1-z \bar{\zeta}}
$$

has finitely many negative squares on $\rho(f)$, the domain of analyticity of $f$. A consequence of this characterization is that, for every point $z \in \rho(f)$, there exist integers $\kappa \geq 0$ and $n_{0}>0$ such that, for every integer $n>n_{0}$, the Hermitian matrix

$$
\mathbf{P}_{n}^{f}(z)=\left[\left.\frac{1}{\ell ! r !} \frac{\partial^{\ell+r}}{\partial z^{\ell} \partial \bar{z}^{r}} \frac{1-|f(z)|^{2}}{1-|z|^{2}}\right|_{z=\zeta}\right]_{\ell, r=0}^{n-1}
$$

which will be referred to as the Schwarz-Pick matrix, has $\kappa$ negative eigenvalues counted with multiplicities. This number $\kappa$ turns out to be equal to the total pole multiplicity of $f$, that is, to the degree of the denominator $b$ in the coprime Krein-Langer representation (1.1). In what follows, we will denote by $v(P)$ the number of negative eigenvalues, counted with multiplicities, of a Hermitian matrix $P$. Straightforward differentiation in (1.2) gives explicit formulas

$$
\begin{aligned}
{\left[\mathbf{P}_{n}^{f}(z)\right]_{\ell, r}=} & \sum_{s=0}^{\min \{\ell, r\}} \frac{(\ell+r-s) !}{(\ell-s) ! s !(r-s) !} \frac{z^{r-s} \bar{z}^{\ell-s}}{\left(1-|z|^{2}\right)^{\ell+r-s+1}} \\
& -\sum_{\alpha=0}^{\ell} \sum_{\beta=0}^{r} \sum_{s=0}^{\min \{\alpha, \beta\}} \frac{(\alpha+\beta-s) !}{(\alpha-s) ! s !(\beta-s) !} \frac{z_{i}^{\beta-s} \bar{z}^{\alpha-s} f_{\ell-\alpha}(z) \overline{f_{r-\beta}(z)}}{\left(1-|z|^{2}\right)^{\alpha+\beta-s+1}}
\end{aligned}
$$

for the entries of $\mathbf{P}_{n}^{f}(z)$ in terms of Taylor coefficients $f_{j}(z):=f^{(j)}(z) / j$ ! and the uniform bound $v\left(\mathbf{P}_{n}^{f}(z)\right) \leq \kappa$ (with actual equality $v\left(\mathbf{P}_{n}^{f}(z)\right)=\kappa$ if $n$ is large enough) eventually leads to a characterization of generalized Schur functions in terms of their Taylor coefficients (see [13]). The objective of this paper is to establish a similar characterization in the boundary context where the ambient point $z$ is moved to the unit circle $\mathbb{T}$, the boundary of $\mathbb{D}$, and where the Taylor coefficients at $z$ are replaced by the boundary limits $f_{j}=\lim _{z \rightarrow t_{0}}\left(f^{(j)}(z) / j\right.$ !). In contrast to the interior case, the boundary limits $f_{j}$ may not exist; however, if the limit $f_{j}$ exists for some $j \in \mathbb{N}$, the limits $f_{k}$ also exist for all $k=0, \ldots, j-1$. We will distinguish two cases: the finite (truncated) problem $\mathbf{P}_{N}$ and the infinite problem $\mathbf{P}_{\infty}$. We will say that $z \in \mathbb{D}$ approaches a boundary point $t_{0} \in \mathbb{T}$ nontangentially and write $z \widehat{\rightarrow} t_{0}$, if $z \in \mathbb{D}$ approaches $t_{0}$ and satisfies $\left|z-t_{0}\right|<\alpha(1-|z|)$ for some fixed $\alpha>1$.

Problem $\mathbf{P}_{N}$. Given a point $t_{0} \in \mathbb{T}$ and given $N<\infty$ complex numbers $f_{0}, \ldots, f_{N}$, find a function $f \in \mathcal{G S}$ which admits the asymptotic expansion

$$
f(z)=f_{0}+f_{1}\left(z-t_{0}\right)+\cdots+f_{N}\left(z-t_{0}\right)^{N}+o\left(\left|z-t_{0}\right|^{N}\right) \quad \text { as } z \widehat{\rightarrow} t_{0} .
$$

Problem $\mathbf{P}_{\infty}$. Given a point $t_{0} \in \mathbb{T}$ and given a complex sequence $\left\{f_{i}\right\}_{i \geq 0}$, find a function $f \in \mathcal{G S}$ which admits the expansion (1.3) for all $N \geq 0$. 
REMARK 1.1. It is known that condition (1.3) holds if and only if the first $N+1$ nontangential derivatives of $f$ exist at $t_{0}$ with values

$$
\lim _{z \rightarrow t_{0}} \frac{f^{(j)}(z)}{j !}=f_{j} \quad \text { for } j=0,1, \ldots, N .
$$

To present the answers to the above problems we first introduce some needed definitions and notation. Given a sequence $\mathbf{f}=\left\{f_{i}\right\}_{i=0}^{N}$ (with $N \leq \infty$ ), we define the lower triangular Toeplitz matrix $\mathbb{U}_{n}^{\mathbf{f}}$ and the Hankel matrix $\mathbb{H}_{n}^{\mathbf{f}}$ by

$$
\mathbb{U}_{n}^{\mathbf{f}}=\left[\begin{array}{cccc}
f_{0} & 0 & \cdots & 0 \\
f_{1} & f_{0} & \ddots & \vdots \\
\vdots & \ddots & \ddots & 0 \\
f_{n-1} & \cdots & f_{1} & f_{0}
\end{array}\right], \quad \mathbb{H}_{n}^{\mathbf{f}}=\left[\begin{array}{cccc}
f_{1} & f_{2} & \cdots & f_{n} \\
f_{2} & f_{3} & \cdots & f_{n+1} \\
\vdots & \vdots & & \vdots \\
f_{n} & f_{n+1} & \cdots & f_{2 n-1}
\end{array}\right]
$$

for every appropriate integer $n \geq 1$ (that is, for every $n \leq N+1$ in the first formula and for every $n \leq(N+1) / 2$ in the second). Given a point $t_{0} \in \mathbb{T}$, we introduce the upper-triangular matrix

$$
\boldsymbol{\Psi}_{n}\left(t_{0}\right)=\left[\begin{array}{cccl}
t_{0} & -t_{0}^{2} & \cdots & (-1)^{n-1}\left(\begin{array}{c}
n-1 \\
0
\end{array}\right) t_{0}^{n} \\
0 & -t_{0}^{3} & \cdots & (-1)^{n-1}\left(\begin{array}{c}
n-1 \\
1
\end{array}\right) t_{0}^{n+1} \\
\vdots & & \ddots & \vdots \\
0 & \cdots & 0 & (-1)^{n-1}\left(\begin{array}{c}
n-1 \\
n-1
\end{array}\right) t_{0}^{2 n-1}
\end{array}\right]
$$

with the entries

$$
\Psi_{j \ell}=\left\{\begin{array}{ll}
0 & \text { if } \quad j>\ell \\
(-1)^{\ell-1}\left(\begin{array}{l}
\ell-1 \\
j-1
\end{array}\right) t_{0}^{\ell+j-1} & \text { if } \quad j \leq \ell
\end{array} \quad(j, \ell=1, \ldots, n),\right.
$$

and finally, for every $n \leq(N+1) / 2$, we introduce the structured matrix

$$
\mathbb{P}_{n}^{\mathbf{f}}=\left[p_{i j}^{\mathbf{f}}\right]_{i, j=1}^{n}=\mathbb{H}_{n}^{\mathbf{f}} \boldsymbol{\Psi}_{n}\left(t_{0}\right) \mathbb{U}_{n}^{\mathbf{f} *}
$$

with the entries (as follows from (1.5)-(1.7))

$$
p_{i j}^{\mathbf{f}}=\sum_{r=1}^{j}\left(\sum_{\ell=1}^{r} f_{i+\ell-1} \Psi_{\ell r}\right) \overline{f_{j-r}}
$$

Since the factors $\boldsymbol{\Psi}_{n}\left(t_{0}\right)$ and $\mathbb{U}_{n}^{\mathbf{f} *}$ in (1.8) are upper-triangular, it follows that $\mathbb{P}_{k}^{\mathbf{f}}$ is the leading submatrix of $\mathbb{P}_{n}^{\mathbf{f}}$ for every $k<n$. Although the matrix $\mathbb{P}_{n}^{\mathbf{f}}$ defined in (1.8) does not have to be Hermitian, it then follows that if it is Hermitian, then the matrices $\mathbb{P}_{k}^{\mathbf{f}}$ 
are Hermitian for all $k<n$. We may thus introduce the quantity $n_{0} \in \mathbb{N} \cup\{\infty\}$ (the size of the maximal Hermitian matrix $\mathbb{P}_{n}^{\mathbf{f}}$ ) by

$$
n_{0}= \begin{cases}0 & \text { if } \mathbb{P}_{1}^{\mathbf{f}}=f_{1} t_{0} \overline{f_{0}} \notin \mathbb{R}, \\ \sup _{1 \leq k \leq(N+1) / 2}\left\{k: \mathbb{P}_{k}^{\mathbf{f}}=\left(\mathbb{P}_{k}^{\mathbf{f}}\right)^{*}\right\} & \text { otherwise, }\end{cases}
$$

with the convention that $n_{0}=\infty$ if the matrices $\mathbb{P}_{k}^{\mathbf{f}}$ are Hermitian for all $k \geq 1$. We also observe that formula (1.9) defines the numbers $p_{i j}^{\mathbf{f}}$ in terms of $\mathbf{f}=\left\{f_{0}, \ldots, f_{N}\right\}$ for every pair of indices $(i, j)$ subject to $i+j \leq N+1$. In particular, if $n \leq N / 2$, one can define via this formula the numbers $p_{n+1, n}^{\mathbf{f}}$ and $p_{n, n+1}^{\mathbf{f}}$ and therefore the number

$$
\gamma_{n}:=t_{0} \cdot\left(p_{n+1, n}^{\mathbf{f}}-\overline{p_{n, n+1}^{\mathbf{f}}}\right) .
$$

The two theorems below are the main results of this paper.

Theorem 1.2. Let $t_{0} \in \mathbb{T}$ and $\mathbf{f}=\left\{f_{0}, \ldots, f_{N}\right\}(1 \leq N<\infty)$ be given. Let the integer $n_{0}$ be defined as in (1.10) and, in case $0<n_{0} \leq N / 2$, let $\gamma_{n_{0}}$ be given by (1.11). The problem $\mathbf{P}_{N}$ has a solution if and only if one of the following holds:

(1) $\left|f_{0}\right|<1$;

(2) $\left|f_{0}\right|=1, n_{0}=(N+1) / 2$;

(3) $\left|f_{0}\right|=1, n_{0}=N / 2, \gamma_{n_{0}} \geq 0$;

(4) $\left|f_{0}\right|=1,0<n_{0}<N / 2, \gamma_{n_{0}}>0$.

Whenever the problem is solvable, it has infinitely many rational solutions.

Theorem 1.3. Let $t_{0} \in \mathbb{T}$ and $\mathbf{f}=\left\{f_{i}\right\}_{i \geq 0}$ be given. Let $n_{0}$ be defined as in (1.10) and, in the case $0<n_{0}<\infty$, let $\gamma_{n_{0}}$ be the number given by (1.11). The problem $\mathbf{P}_{\infty}$ has a solution if and only if one of the following holds:

(1) $\left|f_{0}\right|<1$;

(2) $\left|f_{0}\right|=1, n_{0}<\infty, \gamma_{n_{0}}>0$;

(3) $\left|f_{0}\right|=1, n_{0}=\infty, v\left(\mathbb{P}_{n}^{\mathbf{f}}\right)=\kappa$ for all large $n$ and some $\kappa<\infty$.

The problem may have a unique solution only in case (3).

The Problems $\mathbf{P}_{N}$ and $\mathbf{P}_{\infty}$ have been studied for Schur-class functions $f \in \mathcal{S}$ in [5] and [10], respectively. It was shown that the Problem $\mathbf{P}_{N}$ has infinitely many solutions if and only if either $\left|f_{0}\right|<1$ or the maximal Hermitian matrix $\mathbb{P}_{n_{0}}^{\mathbf{f}}$ is positive definite and one of the cases (2)-(4) in Theorem 1.2 is in force. In contrast to the present setting, the Schur-class problem $\mathbf{P}_{N}$ may have one solution which is the case where the matrix $\mathbb{P}_{n_{0}}^{\mathbf{f}}$ is positive semidefinite (singular) and satisfies certain rank conditions. The infinite problem $\mathbf{P}_{\infty}$ turns out to be solvable in the class $\mathcal{S}$ if and only if either $\left|f_{0}\right|<1$ or the case (2) in Theorem 1.3 is in force with the additional condition $\mathbb{P}_{n_{0}}^{\mathbf{f}}>0$, or the case (3) occurs with the additional condition that $\mathbb{P}_{n}^{\mathbf{f}}$ is positive semidefinite for all $n \geq 1$.

The paper is organized as follows. In Section 2 we present the proof of Theorem 1.2 based on recent results [5] on Schur-class boundary interpolation. The proof of 
Theorem 1.3 is given in Section 3, at the end of which we also discuss the possible determinacy of the problem.

\section{The truncated problem $\mathbf{P}_{N}$}

Since the boundary values of generalized Schur functions cannot exceed one in modulus, the condition $\left|f_{0}\right| \leq 1$ is necessary for the problem $\mathbf{P}_{N}$ to have a solution. On the other hand, the condition $\left|f_{0}\right|<1$ is sufficient: in this case there are infinitely many Schur functions solving the problem (see, for example, [2]). It remains for us to consider a more subtle case where $f_{0}$ is unimodular.

Since every function $f \in \mathcal{G S}$ can be written in the form (1.1) and since the denominator $b \in \mathcal{F} \mathcal{B}$ is analytic on $\overline{\mathbb{D}}$, it is clear that the limits in (1.4) exist if and only if the similar limits for the numerator $s$ exist and satisfy the convolution equalities

$$
\lim _{z \rightarrow t_{0}} \frac{s^{(j)}(z)}{j !}=s_{j}:=\sum_{\ell=0}^{j} b_{\ell} f_{j-\ell} \quad \text { for } j=0, \ldots, N .
$$

Here we have set

$$
b_{j}:=\frac{b^{(j)}\left(t_{0}\right)}{j !}
$$

to be the Taylor coefficients of $b \in \mathcal{F B}$ at the given boundary point $t_{0} \in \mathbb{T}$. For any fixed $b \in \mathcal{F} \mathcal{B}$ we can calculate the sequence $\mathbf{s}=\left\{s_{0}, \ldots, s_{N}\right\}$ via the second equality in (2.1), and if this sequence satisfies the first equality in (2.1) for some $s \in \mathcal{S}$, then the problem $\mathbf{P}_{N}$ has a solution: namely, $f=s / b$. On the other hand, if $\mathbf{f}$ is such that for every $b \in \mathcal{F} \mathcal{B}$, the interpolation conditions (2.1) are satisfied by no Schur function, then the problem $\mathbf{P}_{N}$ has no solutions. This simple idea allows us to reduce the problem $\mathbf{P}_{N}$ to a similar problem for Schur-class functions the answer for which is known [5].

With any $b \in \mathcal{F} \mathcal{B}$ we may associate the matrices $\mathbb{U}_{n}^{b}, \mathbb{H}_{n}^{b}$ and $\mathbb{P}_{n}^{b}$ constructed via formulas (1.5) and (1.8) from the Taylor coefficients (2.2). On the other hand, for the sequence $\mathbf{s}=\left\{s_{0}, \ldots, s_{N}\right\}$ obtained via convolution formulas (2.1) from the given $\mathbf{f}$ and a fixed $b \in \mathcal{F} \mathcal{B}$, we may define the structured matrices

$$
\mathbb{P}_{n}^{\mathbf{s}}=\left[p_{i j}^{\mathbf{s}}\right]_{i, j=1}^{n}=\mathbb{H}_{n}^{\mathbf{s}} \boldsymbol{\Psi}_{n}\left(t_{0}\right) \mathbb{U}_{n}^{\mathbf{s} *}
$$

as in (1.8), with the entries $p_{i j}^{\mathbf{s}}$ defined in the same way as in (1.9). We also may define the numbers

$$
\gamma_{n}^{\mathbf{s}}:=t_{0} \cdot\left(p_{n+1, n}^{\mathbf{s}}-\overline{p_{n, n+1}^{\mathbf{s}}}\right)
$$

for every $n \leq N / 2$ and the integer

$$
n_{0}^{\mathbf{s}}= \begin{cases}0 & \text { if } \mathbb{P}_{1}^{\mathbf{s}}=s_{1} t_{0} \overline{s_{0}} \notin \mathbb{R} \\ \max _{1 \leq k \leq(N+1) / 2}\left\{k: \mathbb{P}_{k}^{\mathbf{s}}=\left(\mathbb{P}_{k}^{\mathbf{s}}\right)^{*}\right\} & \text { otherwise }\end{cases}
$$


Lemma 2.1. Let $b \in \mathcal{F B}$ and let us assume that the two sequences $\mathbf{f}=\left\{f_{0}, \ldots, f_{N}\right\}$ $\left(\left|f_{0}\right|=1\right)$ and $\mathbf{s}=\left\{s_{0}, \ldots, s_{N}\right\}$ are related as in (2.1), Then:

(1) for every $n \geq 1$,

$$
\mathbb{P}_{n}^{b}:=\mathbb{H}_{n}^{b} \Psi_{n}\left(t_{0}\right) \mathbb{U}_{n}^{b *} \geq 0 \quad \text { and } \quad \mathbb{U}_{n}^{b \top} \Psi_{n}\left(t_{0}\right) \mathbb{U}_{n}^{b *}=\Psi_{n}\left(t_{0}\right),
$$

where $\mathbb{U}_{n}^{b \top}$ is the transpose of $\mathbb{U}_{n}^{b}$;

(2) for every $n \leq(N+1) / 2$,

$$
\mathbb{P}_{n}^{\mathbf{S}}:=\mathbb{H}_{n}^{\mathbf{S}} \mathbf{\Psi}_{n}\left(t_{0}\right) \mathbb{U}_{n}^{\mathbf{S} *}=\mathbb{U}_{n}^{\mathbf{f}} \mathbb{P}_{n}^{b} \mathbb{U}_{n}^{\mathbf{f} *}+\mathbb{P}_{n}^{\mathbf{f}}
$$

(3) the integers $n_{0}$ and $n_{0}^{\text {s }}$ defined in (1.10) and (2.4) are equal;

(4) the numbers $\gamma_{n_{0}}$ and $\gamma_{n_{0}}^{\mathbf{s}}$ defined in (1.11) and (2.3) are equal; and

(5) if $b(z)=z^{m}$, then $\mathbb{P}_{n_{0}}^{\text {s }}$ is positive definite for $m$ large enough.

Proof. The proof of the inequality in (2.5) can be found in [4, Lemma 2.1]. The second relation in (2.5) is a consequence of the identity $b(z) \overline{b(1 / \bar{z})} \equiv 1$ (see [8, Theorem 2.5] for details). To prove (2.6) we observe that the convolution equalities (2.1) are equivalent to the matrix equality $\mathbb{U}_{n}^{\mathbf{s}}=\mathbb{U}_{n}^{\mathbf{f}} \mathbb{U}_{n}^{b}$ and imply that

$$
\begin{aligned}
\mathbb{H}_{n}^{\mathbf{s}} & =\left[\begin{array}{cccccc}
0 & & f_{0} & f_{1} & \cdots & f_{n} \\
& . & \vdots & \vdots & . & \vdots \\
f_{0} & \cdots & f_{n-1} & f_{n} & \cdots & f_{2 n-1}
\end{array}\right]\left[\begin{array}{ccc}
b_{n} & \cdots & b_{2 n-1} \\
\vdots & & \vdots \\
b_{1} & \cdots & b_{n} \\
b_{0} & \cdots & b_{n-1} \\
& \ddots & \vdots \\
0 & & b_{0}
\end{array}\right] \\
& =\mathbb{U}_{n}^{\mathbf{f}} \mathbb{H}_{n}^{b}+\mathbb{H}_{n}^{\mathbf{f}} \mathbb{U}_{n}^{b \top} .
\end{aligned}
$$

Making use of the two last identities and of equality (2.5), we get (2.6):

$$
\begin{aligned}
\mathbb{P}_{n}^{\mathbf{s}} & =\mathbb{H}_{n}^{\mathbf{S}} \Psi_{n}\left(t_{0}\right) \mathbb{U}_{n}^{\mathbf{s} *}=\left(\mathbb{U}_{n}^{\mathbf{f}} \mathbb{H}_{n}^{b}+\mathbb{H}_{n}^{\mathbf{f}} \mathbb{U}_{n}^{b \top}\right) \Psi_{n}\left(t_{0}\right) \mathbb{U}_{n}^{b *} \mathbb{U}_{n}^{\mathbf{f} *} \\
& =\mathbb{U}_{n}^{\mathbf{f}} \mathbb{P}_{n}^{b} \mathbb{U}_{n}^{\mathbf{f} *}+\mathbb{H}_{n}^{\mathbf{f}} \Psi_{n}\left(t_{0}\right) \mathbb{U}_{n}^{\mathbf{f} *}=\mathbb{U}_{n}^{\mathbf{f}} \mathbb{P}_{n}^{b} \mathbb{U}_{n}^{\mathbf{f} *}+\mathbb{P}_{n}^{\mathbf{f}} .
\end{aligned}
$$

Since $\mathbb{P}_{n}^{b}$ is Hermitian (by the first relation in (2.5)), it follows from (2.6) that $\mathbb{P}_{n}^{\mathbf{s}}-\mathbb{P}_{n}^{\mathbf{f}}$ is Hermitian for all $n \geq 1$. Statements (3) and (4) are now immediate.

Since $\left|f_{0}\right|=1$, the triangular Toeplitz matrix $\mathbb{U}_{n_{0}}^{\mathbf{f}}$ is invertible, which allows us to write (2.6) (for $n=n_{0}$ ) equivalently as

$$
\left(\mathbb{U}_{n_{0}}^{\mathbf{f}}\right)^{-1} \mathbb{P}_{n_{0}}^{\mathbf{s}}\left(\mathbb{U}_{n_{0}}^{\mathbf{f}}\right)^{-*}=\mathbb{P}_{n_{0}}^{b}+\left(\mathbb{U}_{n_{0}}^{\mathbf{f}}\right)^{-1} \mathbb{P}_{n_{0}}^{\mathbf{f}}\left(\mathbb{U}_{n_{0}}^{\mathbf{f}}\right)^{-*} .
$$

The second term on the right-hand side is completely determined from the given f. Theorem A.1 below gives asymptotics for all eigenvalues of the matrix $\mathbb{P}_{n_{0}}^{b_{m}}$ for $b_{m}(z)=z^{m}$ as $m \rightarrow \infty$. These asymptotics show, in particular, that the minimal eigenvalue of $\mathbb{P}_{n_{0}}^{b_{m}}$ tends to infinity as $m \rightarrow \infty$. Hence, for sufficiently large $m$, the matrix on the left-hand side of (2.7) is positive definite and so is $\mathbb{P}_{n_{0}}^{\mathbf{s}}$, as desired. 
Proof of Theorem 1.2. As we mentioned at the beginning of this section, the problem $\mathbf{P}_{N}$ has infinitely many rational Schur function solutions if $\left|f_{0}\right|<1$ and has no solutions if $\left|f_{0}\right|>1$.

A Carathéodory-Julia-type theorem for generalized Schur functions (see [8, Theorem 4.2]) asserts that whenever a function $f \in \mathcal{G S}$ admits finite boundary limits

$$
f_{0}=\lim _{z \rightarrow t_{0}} f(z) \quad \text { and } \quad f_{1}=\lim _{z \widehat{\rightarrow} t_{0}} f^{\prime}(z)
$$

and $\left|f_{0}\right|=1$, then necessarily $t_{0} f_{1} \bar{f}_{0} \in \mathbb{R}$. Therefore, if $\left|f_{0}\right|=1$ and $n_{0}=0$ (that is, if $\left.t_{0} f_{1} \bar{f}_{0} \notin \mathbb{R}\right)$, the problem $\mathbf{P}_{N}$ has no solutions.

If $\left|f_{0}\right|=1$ and $n_{0}>0$, we fix a finite Blaschke product $b$ and construct the sequence $\mathbf{s}=\left\{s_{0}, \ldots, s_{N}\right\}$ by the convolution formula (2.1). No matter what $b$ we take, we will have $\left|s_{0}\right|=1$ (since $\left.s_{0}=b_{0} f_{0}\right), n_{0}^{\mathbf{s}}=n_{0}$ and $\gamma_{n_{0}}^{\mathbf{s}}=\gamma_{n_{0}}$. By [5, Theorem 2.3], if $\gamma_{n_{0}}^{\mathbf{s}}<0$ (if $n_{0}=N / 2$ ) or if $\gamma_{n_{0}}^{\mathbf{s}} \leq 0$ (if $0<n_{0}<N / 2$ ), there is no Schur-class function $s$ subject to equalities (2.1). Thus, there is no function $f$ of the form (1.1) with $s \in \mathcal{S}$ and $b \in \mathcal{F} \mathcal{B}$ satisfying conditions (1.4). In other words, the problem $\mathbf{P}_{N}$ has no solutions in the following two cases: (1) $n_{0}=N / 2$ and $\gamma_{n_{0}}<0$; (2) $0<n_{0}<N / 2$ and $\gamma_{n_{0}} \leq 0$. On the other hand, upon choosing $b(z)=z^{m}$ with $m$ sufficiently large, we can guarantee that the structured matrix $\mathbb{P}_{n_{0}}^{\mathbf{s}}$ associated with the sequence $\mathbf{s}=\left\{s_{0}, \ldots, s_{N}\right\}$ constructed as in (2.1) is positive definite. In the case when $n_{0}=(N+1) / 2$, this is enough to guarantee the existence of infinitely many rational functions $s \in \mathcal{S}$ satisfying conditions (2.1) (see [2] or [7]). The existence of such functions in the two remaining cases where $n_{0}=N / 2$ and $\gamma_{n_{0}} \geq 0$ or where $0<n_{0}<N / 2$ and $\gamma_{n_{0}}>0$ is guaranteed by [5, Theorem 2.3]. For every such $s$, the function $f(z)=s(z) / z^{m}$ solves the problem $\mathbf{P}_{N}$. This proves the sufficiency of the cases (2)-(4) in Theorem 1.2 which together with the sufficiency of the first case completes the proof of the 'if' part of the theorem. Since we have examined all possible cases and shown that in all other cases the problem has no solutions, the 'only if' part follows.

Remark 2.2. We have shown that whenever the problem $\mathbf{P}_{N}$ has a solution, it has a solution of the form $f(z)=s(z) / z^{m}$ (if $\left|f_{0}\right|<1$, we can let $m=0$ ). On the other hand, the integer $m$ in the latter representation might be far from optimal in the following sense: the problem may have solutions in $\mathcal{G S}$ with the total pole multiplicity $\kappa$ and no solutions of the form $f(z)=s(z) / z^{\kappa}$.

\section{The infinite case}

Comparing the formulations of Theorems 1.2 and 1.3 we can see that only the third case in Theorem 1.3 is essentially infinite. The proof of its sufficiency requires some preliminary work which will be done below. First we will prove the rest of Theorem 1.3. 
3.1. Beginning of the proof of Theorem 1.3. As in the finite case, a necessary condition for the problem $\mathbf{P}_{\infty}$ to have a solution is that $\left|f_{0}\right| \leq 1$. If $\left|f_{0}\right|<1$, then there are infinitely many functions $f \in \mathcal{S}$ subject to conditions (1.4); see [10, Theorem 2.2] for the proof.

It is obvious that if the truncated problem $\mathbf{P}_{N}$ has no solutions for some $N<\infty$, the infinite problem $\mathbf{P}_{\infty}$ has no solutions either. Thus, the absence of solutions in some cases follows from Theorem 1.2. In particular, the problem $\mathbf{P}_{\infty}$ has no solutions if $\left|f_{0}\right|=1$ and $n_{0}=0$. Also, the problem $\mathbf{P}_{\infty}$ has no solutions if $0<n_{0}<\infty$ and $\gamma_{n_{0}} \leq 0$ (recall that $n_{0}$ and $\gamma_{n}$ are defined in (1.10) and (1.11), respectively). On the other hand, if

$$
\left|f_{0}\right|=1, \quad 0<n_{0}<\infty \quad \text { and } \quad \gamma_{n_{0}}>0
$$

then the problem $\mathbf{P}_{\infty}$ has infinitely many solutions of the form $f(z)=s(z) / z^{m}$. Indeed, under assumptions (3.1), we may use the function $b(z)=z^{m}$ to define the infinite sequence $\mathbf{s}=\left\{s_{j}\right\}_{j \geq 0}$ via convolution equalities (2.1). We then have

$$
\left|s_{0}\right|=\left|t_{0}^{m} f_{0}\right|=1, \quad n_{0}^{\mathbf{s}}=n_{0}<\infty, \quad \gamma_{n_{0}}^{\mathbf{s}}=\gamma_{n_{0}}>0, \quad \mathbb{P}_{n_{0}}^{\mathbf{s}}>0
$$

where the first equality is obvious and the next two equalities and the positivity of the structured matrix $\mathbb{P}_{n_{0}}$ for a sufficiently large $m$ follow from Lemma 2.1. By [10, Theorem 1.2], conditions (3.2) are sufficient for the existence of infinitely many functions $s \in \mathcal{S}$ such that

$$
\lim _{z \rightarrow t_{0}} \frac{s^{(j)}(z)}{j !}=s_{j}:=\sum_{\ell=0}^{j} b_{\ell} f_{j-\ell} \quad \text { for } j=0,1, \ldots
$$

For each such $s$, the function $f(z)=s(z) / z^{m}$ solves the problem $\mathbf{P}_{\infty}$.

It remains to consider the case where $\left|f_{0}\right|=1$ and $n_{0}=\infty$; the latter means that the structured matrices $\mathbb{P}_{n}^{\mathbf{f}}$ are Hermitian for all $n \geq 1$. Let us show that in this case uniform boundedness of the negative inertia of matrices $\mathbb{P}_{n}^{\mathbf{f}}$ is necessary for the problem $\mathbf{P}_{N}$ to have a solution. To this end, we first recall a result from [8, Theorem 1.5].

Theorem 3.1. Let $n$ be a positive integer, let $f$ be analytic in a 'neighborhood' $\left\{z \in \mathbb{D}:\left|z-t_{0}\right|<\varepsilon\right\}$ of $t_{0} \in \mathbb{T}$ and let us assume that the nontangential boundary limits $f_{j}=\lim _{z \rightarrow t_{0}}\left(f^{(j)}(z) / j !\right)$ exist for $j=0, \ldots, 2 n-1$ and are such that $\left|f_{0}\right|=1$ and the structured matrix $\mathbb{P}_{n}^{\mathbf{f}}$ constructed from these limits as in (1.8) is Hermitian. Then the Schwarz-Pick matrix $\mathbf{P}_{n}^{f}(z)$ (see (1.2)) converges as $z \widehat{\rightarrow} t_{0}$ and, moreover, $\lim _{z} \widehat{T}_{0} \mathbf{P}_{n}^{f}(z)=\mathbb{P}_{n}^{\mathbf{f}}$.

Note that in Theorem 3.1 the function $f$ is not assumed to be in $\mathcal{G S}$. We now assume that $f$ belongs to $\mathcal{G S}$ and meets conditions (1.4) for all $j \geq 0$. Then all the assumptions in Theorem 3.1 are met and we conclude that

$$
\lim _{z \rightarrow t_{0}} \mathbf{P}_{n}^{f}(z)=\mathbb{P}_{n}^{\mathbf{f}} \quad \text { for all } n \geq 1
$$


Let us denote by $\kappa$ the total pole multiplicity of $f$, that is, the degree of the Blaschke product $b$ in the coprime Krein-Langer representation (1.1) for $f$. By a result of Krein and Langer, the Schwarz-Pick matrix $\mathbf{P}_{n}^{f}(z)$ given by formula (1.2) has at most $\kappa$ negative eigenvalues for every $z \in \rho(f)$ and for every $n \geq 1$. Then we conclude from (3.3) that $v\left(\mathbb{P}_{n}^{\mathbf{f}}\right) \leq v\left(\mathbf{P}_{n}^{f}(z)\right) \leq \kappa$ for all $n \geq 1$.

To complete the proof of Theorem 1.3 it remains to justify the sufficiency of the case (3), which will be done at the end of this section, after some needed preliminaries. For the next three subsections we assume that

$$
\left|f_{0}\right|=1, \quad \mathbb{P}_{n}^{\mathbf{f}}=\mathbb{P}_{n}^{\mathbf{f} *} \quad \text { and } \quad v\left(\mathbb{P}_{n}^{\mathbf{f}}\right) \leq \kappa \quad \text { for all } n \geq 1 .
$$

By the third condition in (3.4), we may assume without loss of generality that $\mathbb{P}_{n}^{\mathbf{f}}$ has exactly $\kappa$ negative eigenvalues if $n$ is large enough:

$$
v\left(\mathbb{P}_{n}^{\mathbf{f}}\right)=\kappa \quad \text { for all } n \geq n_{1} .
$$

For technical convenience we assume that $t_{0} \neq 1$, which we can also do without loss of generality.

3.2. Schur complements and Stein identities. If $\left|f_{0}\right|=1$ and the structured matrix $\mathbb{P}_{n}^{\mathbf{f}}$ is Hermitian, then (see [7, Section 3]) $\mathbb{P}_{n}^{\mathbf{f}}$ satisfies the Stein identity

$$
\mathbb{P}_{n}^{\mathbf{f}}-T_{n} \mathbb{P}_{n}^{\mathbf{f}} T_{n}^{*}=E_{n} E_{n}^{*}-M_{n} M_{n}^{*},
$$

where $T_{n} \in \mathbb{C}^{n \times n}$ and $E_{n}, M_{n} \in \mathbb{C}^{n \times 1}$ are given by

$$
T_{n}=\left[\begin{array}{cccc}
t_{0} & 0 & \cdots & 0 \\
1 & t_{0} & \ddots & \vdots \\
& \ddots & \ddots & 0 \\
0 & & 1 & t_{0}
\end{array}\right], \quad E_{n}=\left[\begin{array}{c}
1 \\
0 \\
\vdots \\
0
\end{array}\right], \quad M_{n}=\left[\begin{array}{c}
f_{0} \\
f_{1} \\
\vdots \\
f_{n-1}
\end{array}\right] .
$$

For every positive integer $d<n$ we write conformal block decompositions

$$
\mathbb{P}_{n}^{\mathbf{f}}=\left[\begin{array}{cc}
\mathbb{P}_{d}^{\mathbf{f}} & B^{*} \\
B & C
\end{array}\right], \quad T_{n}=\left[\begin{array}{cc}
T_{d} & 0 \\
R & T_{n-d}
\end{array}\right], \quad E_{n}=\left[\begin{array}{c}
E_{d} \\
0
\end{array}\right], \quad M_{n}=\left[\begin{array}{c}
M_{d} \\
\widetilde{M}
\end{array}\right],
$$

where $T_{d}, T_{n-d}, E_{d}, M_{d}$ are defined accordingly to (3.7) and where

$$
R=\left[\begin{array}{cccc}
0 & \cdots & 0 & 1 \\
0 & \cdots & 0 & 0 \\
\vdots & & \vdots & \vdots \\
0 & \cdots & 0 & 0
\end{array}\right] \in \mathbb{C}^{(n-d) \times d}, \quad \widetilde{M}=\left[\begin{array}{c}
f_{d} \\
\vdots \\
f_{n-1}
\end{array}\right] \in \mathbb{C}^{(n-d) \times 1}
$$

Substituting block decompositions (3.8) into (3.6) and comparing the corresponding blocks we get the following three equalities:

$$
\begin{aligned}
& \mathbb{P}_{d}^{\mathbf{f}}-T_{d} \mathbb{P}_{d}^{\mathbf{f}} T_{d}^{*}=E_{d} E_{d}^{*}-M_{d} M_{d}^{*}, \\
& B-T_{n-d} B T_{d}^{*}-R P_{d} T_{d}^{*}=-\widetilde{M} M_{d}^{*}, \\
& C-T_{n-d} C T_{n-d}^{*}-R \mathbb{P}_{d}^{\mathbf{f}} R^{*}-T_{n-d} B R^{*}-R B^{*} T_{n-d}^{*}=-\widetilde{M} \widetilde{M}^{*} .
\end{aligned}
$$


Assuming that $\operatorname{det}\left(\mathbb{P}_{d}^{\mathbf{f}}\right) \neq 0$, we define the Schur complement of $\mathbb{P}_{d}^{\mathbf{f}}$ in $\mathbb{P}_{n}^{\mathbf{f}}$ as

$$
\mathbb{S}_{n-d}=C-B\left(\mathbb{P}_{d}^{\mathbf{f}}\right)^{-1} B^{*}
$$

Proposition 3.2. Let $\mathbb{P}_{d}^{\mathbf{f}}$ be an invertible leading submatrix of $\mathbb{P}_{n}^{\mathbf{f}}$. Then its Schur complement $\mathbb{S}_{n-d}$ satisfies the Stein identity

$$
\mathbb{S}_{n-d}-T_{n-d} \mathbb{S}_{n-d} T_{n-d}^{*}=G_{n-d} G_{n-d}^{*}-Y_{n-d} Y_{n-d}^{*}
$$

where $G_{n-d}$ and $Y_{n-d}$ are defined in terms of decompositions (3.8) as

$$
\begin{aligned}
& G_{n-d}=\left(R-\left(I-T_{n-d}\right) B\left(\mathbb{P}_{d}^{\mathbf{f}}\right)^{-1}\right)\left(I-T_{d}\right)^{-1} E_{d}, \\
& Y_{n-d}=\widetilde{M}+\left(R-\left(I-T_{n-d}\right) B\left(\mathbb{P}_{d}^{\mathbf{f}}\right)^{-1}\right)\left(I-T_{d}\right)^{-1} M_{d} .
\end{aligned}
$$

For the proof, it suffices to multiply both sides of (3.6) by $\left[-B\left(\mathbb{P}_{d}^{\mathbf{f}}\right)^{-1} \quad I\right]$ on the left and its adjoint on the right and then to invoke equalities (3.9). Equality (3.10) was proved in [9] for the interior case $t_{0} \in \mathbb{D}$, but the proof does not rely on this assumption, so we refer to [9, Theorem 2.5] for computational details.

Let us denote by $g_{j}$ and $y_{j}$ the entries in the columns (3.11) so that $G_{n-d}=$ $\left[\begin{array}{lll}g_{0} & \cdots & g_{n-d-1}\end{array}\right]^{\top}$ and $Y_{n-d}=\left[\begin{array}{lll}y_{0} & \cdots & y_{n-d-1}\end{array}\right]^{\top}$. Explicit formulas for $g_{j}$ and $y_{j}$ are easily derived from (3.11):

$$
\begin{aligned}
& g_{0}=\left(\mathbf{e}_{d}-\left(1-t_{0}\right) B\left(\mathbb{P}_{d}^{\mathbf{f}}\right)^{-1}\right)\left(I-T_{d}\right)^{-1} E_{d}, \\
& y_{0}=f_{d}+\left(\mathbf{e}_{d}-\left(1-t_{0}\right) B\left(\mathbb{P}_{d}^{\mathbf{f}}\right)^{-1}\right)\left(I-T_{d}\right)^{-1} M_{d},
\end{aligned}
$$

where $\mathbf{e}_{d}=\left[\begin{array}{llll}0 & \cdots & 0 & 1\end{array}\right] \in \mathbb{C}^{1 \times d}$, and

$$
\begin{aligned}
& g_{j}=U_{j}\left(\mathbb{P}_{d}^{\mathbf{f}}\right)^{-1}\left(I-T_{d}\right)^{-1} E_{d}, \\
& \left.y_{j}=f_{j+d}+U_{j}\left(\mathbb{P}_{d}^{\mathbf{f}}\right)^{-1}\right)\left(I-T_{d}\right)^{-1} M_{d} \quad \text { for } j \geq 1,
\end{aligned}
$$

where $U_{j}$ is defined in terms of numbers (1.9) as follows:

$$
U_{j}=\left(t_{0}-1\right) \cdot\left[\begin{array}{lll}
p_{j+d+1,1}^{\mathbf{f}} & \cdots & p_{j+d+1, d}^{\mathbf{f}}
\end{array}\right]+\left[\begin{array}{lll}
p_{j+d, 1}^{\mathbf{f}} & \cdots & p_{j+d, d}^{\mathbf{f}}
\end{array}\right] .
$$

Formulas (3.13) enable us to define $g_{j}$ and $y_{j}$ for every $j \geq 1$. Combined with (1.9), they also show that the numbers $x_{j}$ are completely determined by $t_{0}$ and $f_{0}, f_{1}, \ldots, f_{2 d+j}$ for every fixed $j \geq 0$. Observe that the term $R$ in (3.11) affects only the top entries $g_{0}$ and $y_{0}$ making formulas (3.12) and (3.13) slightly different.

Lemma 3.3. For $g_{0}$ and $y_{0}$ defined as in (3.12), $\left|g_{0}\right|=\left|y_{0}\right| \neq 0$.

Proof. Since $\left|t_{0}\right|=1$, by examining the top-left entries on both sides of (3.10), we get $0=\left|g_{0}\right|^{2}-\left|y_{0}\right|^{2}$ so that $\left|g_{0}\right|=\left|y_{0}\right|$. It will be shown below that

$$
g_{0} E^{*}-y_{0} M^{*}=\left(\mathbf{e}_{d}+B\left(\mathbb{P}_{d}^{\mathbf{f}}\right)^{-1}\left(t_{0} I-T_{d}\right)\right)\left(I-T_{d}\right)^{-1} \mathbb{P}_{d}^{\mathbf{f}}\left(I-T_{d}^{*}\right) .
$$


Since the matrix $\left(I-T_{d}\right)^{-1} \mathbb{P}_{d}^{\mathbf{f}}\left(I-T_{d}^{*}\right)$ is invertible (recall that $\left.t_{0} \neq 1\right)$ and since the rightmost entry in the row-vector $\mathbf{e}_{d}+B\left(\mathbb{P}_{d}^{\mathbf{f}}\right)^{-1}\left(t_{0} I-T_{d}\right)$ is 1 , it follows that the rowvector on the right-hand side of (3.14) is not zero and thus $g_{0}$ and $y_{0}$ cannot both be zeros.

It remains to verify (3.14). We have from (3.12) that

$$
\begin{aligned}
g_{0} E_{d}^{*}-y_{0} M_{d}^{*}=- & f_{d} M_{d}^{*} \\
& +\left(\mathbf{e}_{d}-\left(1-t_{0}\right) B\left(\mathbb{P}_{d}^{\mathbf{f}}\right)^{-1}\right)\left(I-T_{d}\right)^{-1}\left(E_{d} E_{d}^{*}-M_{d} M_{d}^{*}\right) .
\end{aligned}
$$

Due to the first equation in (3.9),

$$
\left(I-T_{d}\right)^{-1}\left(E_{d} E_{d}^{*}-M_{d} M_{d}^{*}\right)=\left(I-T_{d}\right)^{-1} \mathbb{P}_{d}^{\mathbf{f}}\left(1-T_{d}^{*}\right)+\mathbb{P}_{d}^{\mathbf{f}} T_{d}^{*},
$$

while by equating the top rows on both sides of (3.9),

$$
-f_{d} M_{d}^{*}=B-\mathbf{e}_{d} \mathbb{P}_{d}^{\mathbf{f}} T_{d}^{*}-t_{0} B T_{d}^{*} .
$$

Substituting the last two identities into the right-hand side of (3.15) gives

$$
\begin{aligned}
g_{0} E_{d}^{*}-y_{0} M_{d}^{*}=B & -\mathbf{e}_{d} \mathbb{P}_{d}^{\mathbf{f}} T_{d}^{*}-t_{0} B T_{d}^{*} \\
& \quad+\left(\mathbf{e}_{d}-\left(1-t_{0}\right) B\left(\mathbb{P}_{d}^{\mathbf{f}}\right)^{-1}\right)\left(\left(I-T_{d}\right)^{-1} \mathbb{P}_{d}^{\mathbf{f}}\left(1-T_{d}^{*}\right)+\mathbb{P}_{d}^{\mathbf{f}} T_{d}^{*}\right) \\
= & B\left(I-T_{d}^{*}\right)+\mathbf{e}_{d}\left(\mathbb{P}_{d}^{\mathbf{f}}\right)^{-1}\left(I-T_{d}\right)^{-1} \mathbb{P}_{d}^{\mathbf{f}}\left(1-T_{d}^{*}\right) \\
& \quad-\left(1-t_{0}\right) B\left(\mathbb{P}_{d}^{\mathbf{f}}\right)^{-1}\left(I-T_{d}\right)^{-1} \mathbb{P}_{d}^{\mathbf{f}}\left(1-T_{d}^{*}\right),
\end{aligned}
$$

which is clearly equivalent to (3.14). This completes the proof.

The next theorem is the main result of this subsection.

Theorem 3.4. Given $\mathbf{f}=\left\{f_{j}\right\}_{j \geq 0}$, let us assume that conditions (3.4) are met and that the matrix $\mathbb{P}_{d}^{\mathbf{f}}$ is invertible. Define the sequence $\mathbf{x}=\left\{x_{j}\right\}_{j \geq 0}$ as a (unique) solution of the infinite linear system

$$
\sum_{k=0}^{j} x_{k} g_{j-k}=y_{k} \quad \text { for } j=0,1, \ldots,
$$

where $g_{j}$ and $y_{j}$ are defined in (3.12) and (3.13). Then

$$
\left|x_{0}\right|=1, \quad \mathbb{P}_{n}^{\mathbf{x}}=\mathbb{P}_{n}^{\mathbf{x} *} \quad \text { and } \quad v\left(\mathbb{P}_{n}^{\mathbf{x}}\right) \leq \kappa-v\left(\mathbb{P}_{d}^{\mathbf{f}}\right) \quad \text { for all } n \geq 1 .
$$

Proof. Letting $j=0$ in (3.17) we get $x_{0} g_{0}=y_{0}$; therefore, $\left|x_{0}\right|=1$, by Lemma 3.3. For every fixed $n>d$, let $\mathbb{G}_{n-d}$ denote the $(n-d) \times(n-d)$ lower triangular Toeplitz matrix with the leftmost column equal to $G_{n-d}$, so that $\mathbb{G}_{n-d} E_{n-d}=G_{n-d}$. By Lemma 3.3, $g_{0} \neq$ 0 , and hence the matrix $\mathbb{G}_{n-d}$ is invertible. The column $X_{n-d}=\left[\begin{array}{llll}x_{0} & x_{1} & \cdots & x_{n-d-1}\end{array}\right]^{\top}$ satisfies

$$
X_{n-d}=\left[\begin{array}{llll}
x_{0} & x_{1} & \cdots & x_{n-d-1}
\end{array}\right]^{\top}=\mathbb{G}_{n-d}^{-1} Y_{n-d}
$$


due to (3.17). Let us introduce the matrix

$$
\widetilde{P}_{n-d}=\mathbb{G}_{n-d}^{-1} \mathbb{S}_{n-d} \mathbb{G}_{n-d}^{-*}=\mathbb{G}_{n-d}^{-1}\left(C-B\left(\mathbb{P}_{d}^{\mathbf{f}}\right)^{-1} B^{*}\right) \mathbb{G}_{n-d}^{-*} .
$$

By a well-known property of the Schur complement, $v\left(\mathbb{P}_{n}^{\mathbf{f}}\right)=v\left(\mathbb{P}_{d}^{\mathbf{f}}\right)+v\left(\mathbb{S}_{n-d}\right)$. Since $\widetilde{P}_{n-d}$ is congruent to $\mathbb{S}_{n-d}$, it follows that

$$
v\left(\widetilde{P}_{n-d}\right)=v\left(\mathbb{S}_{n-d}\right)=v\left(\mathbb{P}_{n}^{\mathbf{f}}\right)-v\left(\mathbb{P}_{d}^{\mathbf{f}}\right) .
$$

Since the matrix $\mathbb{G}_{n-d}^{-1}$ is lower triangular, it also follows from (3.20) that $\widetilde{P}_{k}$ is a leading principal submatrix of $\widetilde{P}_{n}$ for every $k<n$.

Multiplying both sides of (3.10) by $\mathbb{G}_{n-d}^{-1}$ on the left, by its adjoint on the right, commuting $\mathbb{G}_{n-d}^{-1}$ and $T_{n-d}$ and making use of (3.19) and (3.20), we obtain the Stein identity

$$
\widetilde{P}_{n-d}-T_{n-d} \widetilde{P}_{n-d} T_{n-d}^{*}=E_{n-d} E_{n-d}^{*}-X_{n-d} X_{n-d}^{*} .
$$

Since the latter identity holds for every $n>d$, we conclude that

$$
\widetilde{P}_{n}-T_{n} \widetilde{P}_{n} T_{n}^{*}=E_{n} E_{n}^{*}-X_{n} X_{n}^{*} \quad \text { for all } n \geq 1 .
$$

By [6, Theorem 10.5], a necessary and sufficient condition for the Stein equation

$$
A-T_{n} A T_{n}^{*}=E_{n} E_{n}^{*}-X_{n} X_{n}^{*}
$$

to have a solution $A \in \mathbb{C}^{n \times n}$ is that

$$
\mathbb{U}_{n}^{\mathbf{X} \top} \Psi_{n}\left(t_{0}\right) \mathbb{U}_{n}^{\mathbf{X} *}=\Psi_{n}\left(t_{0}\right),
$$

where $\mathbb{U}_{n}^{\mathbf{x}}$ and $\Psi_{n}\left(t_{0}\right)$ are defined via formulas (1.5) and (1.6). Thus, equality (3.23) holds for all $n \geq 1$. By [8, Theorem 2.5], the double-sized equality

$$
\mathbb{U}_{2 n}^{\mathbf{X} \top} \Psi_{2 n}\left(t_{0}\right) \mathbb{U}_{2 n}^{\mathbf{X} *}=\Psi_{2 n}\left(t_{0}\right)
$$

guarantees that the structured matrix $\mathbb{P}_{n}^{\mathbf{X}}$ is Hermitian (which proves the second equality in (3.18)) and, therefore, it satisfies the same Stein equation (3.22) as $\widetilde{P}_{n}$. It is known that the Stein equation (3.22) uniquely determines the entries $a_{i j}$ (for $2 \leq i+j \leq n$ ) of its solution $A=\left[a_{i j}\right]_{i, j=1}^{n}$ (see [6, p. 77]). Therefore, the $(i, j)$ entry in $\widetilde{P}_{n}$ is equal to the corresponding entry in $\mathbb{P}_{n}^{\mathbf{x}}$ for all $(i, j)$ subject to $2 \leq i+j \leq n$. Since $\widetilde{P}_{n}$ and $\mathbb{P}_{n}^{\mathbf{x}}$ are leading submatrices of respectively $\widetilde{P}_{m}$ and $\mathbb{P}_{m}^{\mathbf{x}}$ for all $m>n$, we may increase $n$ to conclude that $\mathbb{P}_{n}^{\mathbf{x}}=\widetilde{P}_{n}$ for all $n \geq 1$. Now the last relation in (3.18) follows from (3.21).

By our assumption (3.5), $v\left(\mathbb{P}_{d}^{\mathbf{f}}\right)=\kappa$ if $d$ is large enough. For such $d$ we would conclude from the third relation in (3.18) that $v\left(\mathbb{P}_{n}^{\mathbf{X}}\right)=0$, that is, that the matrix $\mathbb{P}_{n}^{\mathbf{X}}$ is positive semidefinite for all $n \geq 1$. The question is whether there exists an invertible matrix $\mathbb{P}_{d}^{\mathbf{f}}$ which captures the maximally possible negative inertia. The next lemma shows that such $d$ always exists. 
Lemma 3.5. Let us assume that conditions (3.4) and (3.5) are in force. Then there exists an integer $d \geq 1$ such that $\mathbb{P}_{d}^{\mathbf{f}}$ is invertible and $v\left(\mathbb{P}_{d}^{\mathbf{f}}\right)=\kappa$.

Proof. Let us define $\widetilde{n}=\sup \left\{n \in \mathbb{N}: \operatorname{det} \mathbb{P}_{n}^{\mathbf{f}} \neq 0\right\}$. If $\widetilde{n}=\infty$, the statement of the lemma is obvious due to (3.5). If $\widetilde{n}<\infty$, we can take $d=\widetilde{n}$, since for this choice of $d$, as we will show below, $v\left(\mathbb{P}_{d}^{\mathbf{f}}\right)=\kappa$. Indeed, since $\mathbb{P}_{d}^{\mathbf{f}}$ is invertible, we can define the sequence $\mathbf{x}=\left\{x_{j}\right\}_{j \geq 0}$ as in (3.17). By the proof of Theorem 3.4, the structured matrix $\mathbb{P}_{m}^{\mathbf{x}}$ is congruent to the Schur complement $\mathbb{S}_{m}$ of $\mathbb{P}_{d}^{\mathbf{f}}$ in $\mathbb{P}_{d+m}^{\mathbf{f}}$ for every $m \geq 1$. The definition of $d=\widetilde{n}$ tells us that det $\mathbb{P}_{d+m}^{\mathbf{f}}=0$ for every $m \geq 1$ so that $\mathbb{P}_{m}^{\mathbf{x}}$ is singular for every $m \geq 1$. Due to the structure (1.8) of $\mathbb{P}_{m}^{\mathbf{x}}=\mathbb{H}_{m}^{\mathbf{X}} \boldsymbol{\Psi}_{m}\left(t_{0}\right) \mathbb{U}_{m}^{\mathbf{X} *}$ and since the matrices $\boldsymbol{\Psi}_{m}\left(t_{0}\right)$ and $\mathbb{U}_{m}^{\mathbf{X} *}$ are invertible (recall that $\left|x_{0}\right|=1$, by Theorem 3.4), it follows that the Hankel matrix $\mathbb{H} \mathbb{H}_{m}^{\mathbf{X}}=\left[x_{i+j-1}\right]_{i, j=1}^{m}$ is singular for every $m \geq 1$. The latter implies that $x_{j}=0$ for every $j \geq 1$. Therefore, $\mathbb{P}_{m}^{\mathbf{x}}=0$ and hence $v\left(\mathbb{P}_{d+m}^{\mathbf{f}}\right)=v\left(\mathbb{P}_{d}^{\mathbf{f}}\right)+v\left(\mathbb{P}_{m}^{\mathbf{x}}\right)=v\left(\mathbb{P}_{d}^{\mathbf{f}}\right)$ for all $m \geq 1$. Combining this with (3.5) leads us to $v\left(\mathbb{P}_{d}^{\mathbf{f}}\right)=\kappa$, which completes the proof of the lemma.

3.3. The matrix-function $\boldsymbol{\Theta}$. Still assuming that $t_{0} \neq 1,\left|f_{0}\right|=1$ and the matrix $\mathbb{P}_{d}^{\mathbf{f}}$ is Hermitian and invertible, let us introduce the $2 \times 2$ matrix-valued function

$$
\Theta(z)=I+(z-1)\left[\begin{array}{c}
E_{d}^{*} \\
M_{d}^{*}
\end{array}\right]\left(I-z T_{d}^{*}\right)^{-1}\left(\mathbb{P}_{d}^{\mathbf{f}}\right)^{-1}\left(I-T_{d}\right)^{-1}\left[\begin{array}{ll}
E_{d} & -M_{d}
\end{array}\right]
$$

and let

$$
\widetilde{\Theta}(z)=\left[\begin{array}{cc}
\widetilde{\theta}_{11}(z) & \widetilde{\theta}_{12}(z) \\
\widetilde{\theta}_{21}(z) & \widetilde{\theta}_{22}(z)
\end{array}\right]:=\left(z-t_{0}\right)^{d} \cdot \Theta(z) .
$$

Since $\left(I-z T_{d}^{*}\right)^{-1}$ is the upper-triangular Toeplitz matrix with the top row equal to

$$
\left[\begin{array}{llll}
\left(1-z \bar{t}_{0}\right)^{-1} & z\left(1-z \bar{t}_{0}\right)^{-2} & \cdots & z^{d-1}\left(1-z \bar{t}_{0}\right)^{-d}
\end{array}\right]
$$

the function $\Theta$ is rational with the only pole of multiplicity $d$ at $t_{0}$, whereas $\widetilde{\Theta}$ is a matrix polynomial. It is not hard to see from (3.24), (3.25) and (3.7) that

$$
\widetilde{\Theta}\left(t_{0}\right)=(-1)^{d} t_{0}^{2 d-1}\left(t_{0}-1\right)\left[\begin{array}{l}
1 \\
\bar{f}_{0}
\end{array}\right] \mathbf{e}_{d}\left(\mathbb{P}_{d}^{\mathbf{f}}\right)^{-1}\left(I-T_{d}\right)^{-1}\left[\begin{array}{ll}
E_{d} & -M_{d}
\end{array}\right],
$$

where $\mathbf{e}_{d}$ is the row-vector introduced just above formula (3.13). The next lemma establishes several equalities needed for the subsequent analysis.

Lemma 3.6. Let $\Theta$ and $\widetilde{\Theta}$ be defined as in (3.24), (3.25) and let $G_{n-d}, Y_{n-d}$ be the columns given in (3.11) with the top entries $g_{0}, y_{0}$ displayed in (3.12). Then

$$
\widetilde{\theta}_{21}\left(t_{0}\right) \bar{g}_{0}+\widetilde{\theta}_{22}\left(t_{0}\right) \bar{y}_{0}=\frac{(-1)^{d} t_{0}^{2 d-1}\left(t_{0}-1\right) \bar{f}_{0}}{1-\bar{t}_{0}} .
$$

Furthermore, if for some $n>d$, the numbers $f_{2 d}, \ldots, f_{2 n-1}$ are such that the matrix $\mathbb{P}_{n}^{\mathbf{f}}$ is Hermitian, then

$$
\left(z I-T_{n}\right)^{-1}\left[\begin{array}{ll}
E_{n} & -M_{n}
\end{array}\right] \Theta(z)=\left[\begin{array}{c}
0 \\
\left(z I-T_{n-d}\right)^{-1}\left[\begin{array}{ll}
G_{n-d} & -Y_{n-d}
\end{array}\right]
\end{array}\right]+\Phi(z),
$$


where $\Phi(z)$ is defined in terms of decompositions (3.8) as follows:

$$
\Phi(z)=\left[\begin{array}{c}
\mathbb{P}_{d}^{\mathbf{f}} \\
B
\end{array}\right]\left(I-z T_{d}^{*}\right)^{-1}\left(\mathbb{P}_{d}^{\mathbf{f}}\right)^{-1}\left(I-T_{d}\right)^{-1}\left[\begin{array}{ll}
E_{d} & -M_{d}
\end{array}\right] .
$$

Finally, det $\widetilde{\Theta}(z)=\left(z-t_{0}\right)^{2 d}$ for all $z \in \mathbb{C}$ and $\left|\widetilde{\theta}_{21}\left(t_{0}\right)\right|=\left|\widetilde{\theta}_{22}\left(t_{0}\right)\right| \neq 0$.

Proof. It follows from (3.26) that

$$
\begin{aligned}
\widetilde{\theta}_{21}\left(t_{0}\right) \bar{g}_{0}+\widetilde{\theta}_{22}\left(t_{0}\right) \bar{y}_{0}=(-1)^{d} t_{0}^{2 d-1}\left(t_{0}-1\right) \bar{f}_{0} \mathbf{e}_{d}\left(\mathbb{P}_{d}^{\mathbf{f}}\right)^{-1}\left(I-T_{d}\right)^{-1} \\
\times\left(E_{d} \bar{g}_{0}-M_{d} \bar{y}_{0}\right) .
\end{aligned}
$$

Taking adjoints on both sides of the second equality in (3.9) we see that

$$
\begin{aligned}
& \mathbf{e}_{d}\left(\mathbb{P}_{d}^{\mathbf{f}}\right)^{-1}\left(I-T_{d}\right)^{-1}\left(E_{d} \bar{g}_{0}-M_{d} \bar{y}_{0}\right) \\
& \quad=\mathbf{e}_{d}\left(I-T_{d}^{*}\right)^{-1}\left\{\mathbf{e}_{d}^{*}+\left(\left(\bar{t}_{0}-T_{d}^{*}\right) \mathbb{P}_{d}^{\mathbf{f}}\right)^{-1} B^{*}\right\}=\frac{1}{1-\bar{t}_{0}},
\end{aligned}
$$

where the last equality holds true since $\mathbf{e}_{d}\left(I-T_{d}^{*}\right)^{-1}\left(\bar{t}_{0}-T_{d}^{*}\right)=0$. Substituting (3.31) into (3.30) gives (3.27).

Since $\left|f_{0}\right|=1$ and since $P_{n}^{\mathbf{f}}$ is Hermitian, it follows that the Stein identity (3.6) holds (we again refer to [7] for the proof) which is equivalent to the three identities in (3.9). To verify (3.28), it suffices to plug in the formula (3.24) for $\Theta$, the decompositions (3.8) and the conformal decomposition

$$
\left(z I-T_{n}\right)^{-1}=\left[\begin{array}{cc}
\left(z I-T_{d}\right)^{-1} & 0 \\
\left(z I-T_{n-d}\right)^{-1} R\left(z I-T_{d}\right)^{-1} & \left(z I-T_{n-d}\right)^{-1}
\end{array}\right]
$$

into the left-hand side of (3.28) and then to invoke the two top identities in (3.9). The calculations are straightforward and will be omitted.

To prove the formula for $\operatorname{det} \widetilde{\Theta}(z)$ we use the well-known determinantal equality $\operatorname{det}(I+A B)=\operatorname{det}(I+B A)$ along with the explicit formula (3.24) and the Stein identity from (3.9):

$$
\begin{aligned}
\operatorname{det} \Theta(z) & =\operatorname{det}\left(I+(z-1)\left(I-z T_{d}^{*}\right)^{-1}\left(\mathbb{P}_{d}^{\mathbf{f}}\right)^{-1}\left(I-T_{d}\right)^{-1}\left[\begin{array}{ll}
E_{d} & -M_{d}
\end{array}\right]\left[\begin{array}{c}
E_{d}^{*} \\
M_{d}^{*}
\end{array}\right]\right) \\
& =\operatorname{det}\left(I+(z-1)\left(I-z T_{d}^{*}\right)^{-1}\left(\mathbb{P}_{d}^{\mathbf{f}}\right)^{-1}\left(I-T_{d}\right)^{-1}\left(\mathbb{P}_{d}^{\mathbf{f}}-T_{d} \mathbb{P}_{d}^{\mathbf{f}} T_{d}^{*}\right)\right) \\
& =\operatorname{det}\left(\left(I-z T_{d}^{*}\right)^{-1}\left(\mathbb{P}_{d}^{\mathbf{f}}\right)^{-1}\left(I-T_{d}\right)^{-1}\left(z I-T_{d}\right) \mathbb{P}_{d}^{\mathbf{f}}\left(I-T_{d}^{*}\right)\right) \\
& =\frac{\operatorname{det}\left(z I-T_{d}\right) \cdot \operatorname{det}\left(I-T_{d}^{*}\right)}{\operatorname{det}\left(I-z T_{d}^{*}\right) \cdot \operatorname{det}\left(I-T_{d}\right)}=1 \quad\left(z \neq t_{0}\right),
\end{aligned}
$$

where the last equality follows from the special structure (3.7) of $T_{d}$. The desired formula det $\widetilde{\Theta}(z)=\left(z-t_{0}\right)^{2 d}$ follows from (3.25). 
Finally, we have from (3.26) and (3.16) that

$$
\begin{aligned}
& \left.\widetilde{\theta}_{21}\left(t_{0}\right)\right|^{2}-\left|\widetilde{\theta}_{21}\left(t_{0}\right)\right|^{2} \\
& \quad=\left|t_{0}-1\right|^{2} \cdot \mathbf{e}_{d}\left(\mathbb{P}_{d}^{\mathbf{f}}\right)^{-1}\left(I-T_{d}\right)^{-1}\left(E_{d} E_{d}^{*}-M_{d} M_{d}^{*}\right)\left(I-T_{d}^{*}\right)^{-1}\left(\mathbb{P}_{d}^{\mathbf{f}}\right)^{-1} \mathbf{e}_{d}^{*} \\
& \quad=\left|t_{0}-1\right|^{2} \mathbf{e}_{d}\left(\left(\mathbb{P}_{d}^{\mathbf{f}}\right)^{-1}\left(I-T_{d}\right)^{-1}+T_{d}^{*}\left(I-T_{d}^{*}\right)^{-1}\left(\mathbb{P}_{d}^{\mathbf{f}}\right)^{-1}\right) \mathbf{e}_{d}^{*},
\end{aligned}
$$

where the first equality follows from (3.26) and the second equality is a consequence of (3.16). Due to the special form of $T_{d}$ and $\mathbf{e}_{d}$,

$$
\left(I-T_{d}\right)^{-1} \mathbf{e}_{d}^{*}=\frac{1}{1-t_{0}} \mathbf{e}_{d}^{*}, \quad \mathbf{e}_{d} T_{d}^{*}\left(I-T_{d}^{*}\right)^{-1}=\frac{\bar{t}_{0}}{1-\bar{t}_{0}} \mathbf{e}_{d}=\frac{1}{t_{0}-1} \mathbf{e}_{d},
$$

which being substituted in (3.32) gives $\left|\widetilde{\theta}_{21}\left(t_{0}\right)\right|^{2}-\left|\widetilde{\theta}_{21}\left(t_{0}\right)\right|^{2}=0$. Since by $(3.27), \widetilde{\theta}_{21}\left(t_{0}\right)$ and $\widetilde{\theta}_{22}\left(t_{0}\right)$ cannot both be equal to zero, it follows that $\left|\widetilde{\theta}_{21}\left(t_{0}\right)\right|=\left|\widetilde{\theta}_{22}\left(t_{0}\right)\right| \neq 0$, which completes the proof.

3.4. The Schur reduction. The idea going back to Schur [14] is to reduce a given interpolation problem to a similar one but with fewer interpolation conditions.

Theorem 3.7. Let us assume that $t_{0} \neq 1,\left|f_{0}\right|=1$ and that the matrix $\mathbb{P}_{d}^{\mathbf{f}}=\mathbb{P}_{d}^{\mathbf{f} *}$ is invertible. Let $\widetilde{\Theta}$ be defined as in (3.25) and (3.24). Then a function $f$ belongs to $G \mathcal{S}$, has $v\left(\mathbb{P}_{d}^{\mathbf{f}}\right)$ poles inside $\mathbb{D}$ and admits the boundary asymptotic

$$
f(z)=f_{0}+f_{1}\left(z-t_{0}\right)+\cdots+f_{2 d-1}\left(z-t_{0}\right)^{2 d-1}+O\left(\left|z-t_{0}\right|^{2 d}\right)
$$

as $z \widehat{\rightarrow} t_{0}$ if and only if it is of the form

$$
f=\frac{\widetilde{\theta}_{11} h+\widetilde{\theta}_{12}}{\widetilde{\theta}_{21} h+\widetilde{\theta}_{22}}
$$

for some $h \in \mathcal{S}$ such that the boundary limit $h_{0}=\lim _{z \rightarrow t_{0}} h(z)$ either does not exist or satisfies

$$
\widetilde{\theta}_{21}\left(t_{0}\right) h_{0}+\widetilde{\theta}_{22}\left(t_{0}\right) \neq 0 \text {. }
$$

The proof is given in [2] (see also [1, 3]) for rational functions (in which case $h_{0}=$ $h\left(t_{0}\right)$ always exists and the nontangential approach to the boundary can be replaced by evaluation at $t_{0}$ ), but all the arguments go through in the general meromorphic setting. We remark, however, that in the general setting condition (3.33) is not equivalent to the condition with the additional term of the form $o\left(\left|z-t_{0}\right|^{2 d-1}\right)$ as in the problem $\mathbf{P}_{2 d-1}$.

We now use Theorem 3.7 to carry out the Schur reduction.

Theorem 3.8. Given $\mathbf{f}=\left\{f_{j}\right\}_{j \geq 0}$, let us assume that conditions (3.4) and (3.5) are met and that the matrix $\mathbb{P}_{d}^{\mathbf{f}}$ is invertible. Let $\mathbf{x}=\left\{x_{j}\right\}_{j \geq 0}$ be the sequence defined in (3.17). 
A function $f$ is a solution to the problem $\mathbf{P}_{\infty}$ and has $\kappa$ poles inside $\mathbb{D}$ if and only if it is of the form (3.34) for some $h \in \mathcal{S}$ such that

$$
\lim _{z \rightarrow t_{0}} \frac{h^{(j)}(z)}{j !}=x_{j} \quad \text { for all } j \geq 0
$$

Proof. Let us assume that $f$ is a solution to the problem $\mathbf{P}_{\infty}$ and has $\kappa$ poles inside D. In particular, $f$ satisfies condition (3.33) and, therefore, it can be represented in the form (3.34) for some Schur function $h \in \mathcal{S}$. Since $\widetilde{\theta}_{22}\left(t_{0}\right) \neq 0$ (by Lemma 3.6), the Schur function $s \equiv 0$ meets condition (3.35) and, therefore, the rational function $\mathbf{a}=\left(\widetilde{\theta}_{11} s+\widetilde{\theta}_{12}\right) /\left(\widetilde{\theta}_{21} s+\widetilde{\theta}_{22}\right)=\widetilde{\theta}_{12} / \widetilde{\theta}_{22}$ satisfies condition (3.33), by Theorem 3.7. We have

$$
f=\frac{\widetilde{\theta}_{11} h+\widetilde{\theta}_{12}}{\widetilde{\theta}_{21} h+\widetilde{\theta}_{22}}=\mathbf{a}+\frac{\operatorname{det} \widetilde{\Theta} \cdot h}{\widetilde{\theta}_{22}\left(\widetilde{\theta}_{21} h+\widetilde{\theta}_{22}\right)} .
$$

Since both $f$ and a satisfy the same asymptotic equality (3.33) and since $f$ solves in addition the problem $\mathbf{P}_{\infty}$, it follows that

$$
f(z)-\mathbf{a}(z)=f_{2 d}-\frac{\mathbf{a}^{(2 d)}\left(t_{0}\right)}{(2 d) !}+O\left(\left|z-t_{0}\right|^{2 d}\right) .
$$

Since det $\widetilde{\Theta}(z)=\left(z-t_{0}\right)^{2 d}$ (by Lemma 3.6), we may conclude from the two latter equalities that the limit $h_{0}=\lim _{z} \widehat{\rightarrow t}_{0} h(z)$ exists and satisfies the equality

$$
f_{2 d}-\frac{\mathbf{a}^{(2 d)}\left(t_{0}\right)}{(2 d) !}=\frac{h_{0}}{\widetilde{\theta}_{22}\left(t_{0}\right)\left(\widetilde{\theta}_{21} h_{0}+\widetilde{\theta}_{22}\left(t_{0}\right)\right)} .
$$

By Theorem 3.7, $h_{0}$ satisfies inequality (3.35) (this can be derived directly from (3.37)). Observe that

$$
f(z)-\sum_{j=0}^{n-1} f_{j}\left(z-t_{0}\right)^{j}=\left(z-t_{0}\right)^{n} \cdot \mathbf{e}_{n}\left(z I-T_{n}\right)^{-1}\left[\begin{array}{ll}
E_{n} & -M_{n}
\end{array}\right]\left[\begin{array}{c}
f(z) \\
1
\end{array}\right]
$$

where $\mathbf{e}_{n}=\left[\begin{array}{llll}0 & \cdots & 0 & 1\end{array}\right]^{\top} \in \mathbb{C}^{1 \times n}$. Also observe that equality (3.34) can be written as

$$
\left[\begin{array}{l}
f \\
1
\end{array}\right]=\widetilde{\Theta}\left[\begin{array}{l}
h \\
1
\end{array}\right] \cdot \mathbf{q} \quad \text { where } \mathbf{q}=\widetilde{\theta}_{21} h+\widetilde{\theta}_{22}
$$


Substituting (3.39) into (3.38) and making use of (3.29) gives

$$
\begin{aligned}
& f(z)-\sum_{j=0}^{n-1} f_{j}\left(z-t_{0}\right)^{j} \\
& =\left(z-t_{0}\right)^{n} \cdot \mathbf{e}_{n}\left(z I-T_{n}\right)^{-1}\left[\begin{array}{ll}
E_{n} & -M_{n}
\end{array}\right] \widetilde{\Theta}(z)\left[\begin{array}{c}
h(z) \\
1
\end{array}\right] \mathbf{q}(z)
\end{aligned}
$$

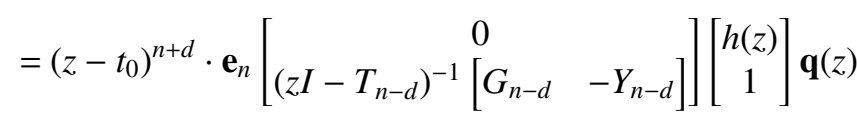

$$
\begin{aligned}
& +\left(z-t_{0}\right)^{n+d} \cdot \mathbf{e}_{n} \Phi(z)\left[\begin{array}{c}
h(z) \\
1
\end{array}\right] \mathbf{q}(z) \\
& =\left(z-t_{0}\right)^{n+d} \cdot \mathbf{e}_{n-d}\left(z I-T_{n-d}\right)^{-1}\left(G_{n-d} h(z)-Y_{n-d}\right) \mathbf{q}(z) \\
& +\left(z-t_{0}\right)^{n+d} \cdot \mathbf{e}_{n} \Phi(z)\left[\begin{array}{c}
h(z) \\
1
\end{array}\right] \mathbf{q}(z)
\end{aligned}
$$

Since $f$ solves the problem $\mathbf{P}_{\infty}$,

$$
f(z)-\sum_{j=0}^{n-1} f_{j}\left(z-t_{0}\right)^{j}=O\left(\left|z-t_{0}\right|^{n}\right)
$$

for all $n \geq 1$ as $z \widehat{\rightarrow} t_{0}$. Since $h$ is uniformly bounded on $\mathbb{D}$, it follows from (3.35) and formula (3.29) for $\Phi$ that

$$
\left(z-t_{0}\right)^{n+d} \cdot \mathbf{e}_{n} \Phi(z)\left[\begin{array}{c}
h(z) \\
1
\end{array}\right] \mathbf{q}(z)=O\left(\left|z-t_{0}\right|^{n}\right) \quad \text { for all } n \geq 1 .
$$

Now we conclude from (3.40) that

$$
\left(z-t_{0}\right)^{d} \cdot \mathbf{e}_{n-d}\left(z I-T_{n-d}\right)^{-1}\left(G_{n-d} h(z)-Y_{n-d}\right)=O(1)
$$

for all $n>d$. Of course, the latter relation is trivial and contains no information for $n=d+1, \ldots, 2 d$. For $n>2 d$, let us multiply both sides of (3.42) by $\left(z-t_{0}\right)^{n-2 d}$ and take into account the structure of $T_{n-d}$ to get

$$
\left[\begin{array}{cccc}
1 & z-t_{0} & \cdots & \left(z-t_{0}\right)^{n-2 d-1}
\end{array}\right]\left(G_{n-d} h(z)-Y_{n-d}\right)=O\left(\left|z-t_{0}\right|^{n-2 d}\right)
$$

or equivalently (in terms of the entries $g_{j}$ and $y_{j}$ defined in (3.12) and (3.13)) as

$$
h(z) \cdot \sum_{j=0}^{n-2 d-1} g_{j}\left(z-t_{0}\right)^{j}-\sum_{j=0}^{n-2 d-1} y_{j}\left(z-t_{0}\right)^{j}=O\left(\left|z-t_{0}\right|^{n-2 d}\right) .
$$

Due to convolution relations (3.17) and since $g_{0} \neq 0$, the latter equality is equivalent to

$$
h(z)-\sum_{j=0}^{n-2 d-1} x_{j}\left(z-t_{0}\right)^{j}=O\left(\left|z-t_{0}\right|^{n-2 d}\right)
$$


which, in turn, implies equalities (3.36) for $j=0, \ldots, n-2 d-1$. Since $n$ can be chosen arbitrarily large, we get equalities (3.36) for all $j \geq 0$.

Conversely, let us assume that $h$ is a Schur-class function satisfying conditions (3.36). Since $x_{0}=y_{0} / g_{0}$ by the first equation in (3.17) and since $x_{0}$ is unimodular (by Lemma 3.3), it follows from (3.27) that

$$
\widetilde{\theta}_{21}\left(t_{0}\right) x_{0}+\widetilde{\theta}_{22}\left(t_{0}\right)=\frac{1}{\bar{y}_{0}}\left(\widetilde{\theta}_{21}\left(t_{0}\right) \bar{g}_{0}+\widetilde{\theta}_{22}\left(t_{0}\right) \bar{y}_{0}\right)=\frac{(-1)^{d} t_{0}^{2 d-1}\left(t_{0}-1\right) \bar{f}_{0}}{\bar{y}_{0}\left(1-\bar{t}_{0}\right)}
$$

and thus condition (3.35) is satisfied. By Theorem 3.7, the function $f$ constructed from $h$ by formula (3.34) has $\kappa$ poles inside $\mathbb{D}$ and satisfies (3.33), that is, the requested boundary derivatives $f_{n}$ at $t_{0}$ for $n=0, \ldots, 2 d$. For $n>2 d$ we use calculation (3.40) to conclude that equalities (3.36) for $j=0, \ldots, n-2 d-1$ for $h$ imply the asymptotic equality (3.41) for $f$. Letting $n$ go to infinity we then conclude that $f$ is a solution to the problem $\mathbf{P}_{\infty}$.

3.5. Completion of the proof of Theorem 1.3. We complete the proof of Theorem 1.3 by demonstrating the sufficiency of conditions (3.4) and (3.5). By Lemma 3.5, we can find $d \geq 1$ so that $\mathbb{P}_{d}^{\mathbf{f}}$ is invertible and $v\left(\mathbb{P}_{d}^{\mathbf{f}}\right)=\kappa$. Let $\mathbf{x}$ be the sequence defined in (3.17). By Theorem 3.8, the problem $\mathbf{P}_{\infty}$ has a solution $f \in \mathcal{G S}$ with $\kappa$ poles inside $\mathbb{D}$ if and only if there exists a Schur function $h \in \mathcal{S}$ subject to interpolation conditions (3.36). Since the structured matrices $\mathbb{P}_{n}^{\mathbf{x}}$ are positive semidefinite for all $n \geq 0$ (by Theorem 3.4 and since $v\left(\mathbb{P}_{d}^{\mathbf{f}}\right)=\kappa$ ), such a function $h$ does exist (see, for example, [11]). Substituting this $h$ into (3.34) results in a solution $f$ to the problem $\mathbf{P}_{\infty}$.

3.6. Concluding remarks. Since in cases (1) and (2) the problem $\mathbf{P}_{\infty}$ is indeterminate, the last statement in Theorem 1.3 need not be proved. However, we will show that in case (3) the problem $\mathbf{P}_{\infty}$ indeed may be determinate.

Let us consider the subcase of (3) where there exists the maximal invertible structured matrix $\mathbb{P}_{d}^{\mathbf{x}}$. Then for the associated sequence $\mathbf{x}=\left\{x_{j}\right\}$ we have $x_{j}=0$ for all $j \geq 1$ and the only Schur function $h$ satisfying conditions (3.36) is a unimodular constant function $h \equiv x_{0}$. Substituting this $h$ into (3.34) we get a solution $f$ to the problem $\mathbf{P}_{\infty}$. This $f$ is rational and has $\kappa$ poles inside $\mathbb{D}$ (by Theorem 3.7). It is not hard to show that deg $f=d$ and that $f$ is unimodular on $\mathbb{T}$. Therefore, $f$ is the ratio of two Blaschke products of the respective degrees $d-\kappa$ and $\kappa$. So far, we have shown that $\mathbf{P}_{\infty}$ has a unique solution $f \in \mathcal{G S}$ with $\kappa$ poles inside $\mathbb{D}$. Let us assume that $\widetilde{f} \in \mathcal{G} \mathcal{S}$ is another solution to the problem $\mathbf{P}_{\infty}$. Take it in the form of (1.1), that is, $\widetilde{f}=s / b$ for some $s \in \mathcal{S}$ and $b \in \mathcal{F} \mathcal{B}$. Then the associated structured matrices $\mathbb{P}_{n}^{\mathbf{f}}, \mathbb{P}_{n}^{s}$ and $\mathbb{P}_{n}^{b}$ are related as in (2.6) for all $n \geq 1$. Since rank $\mathbb{P}_{n}^{b}=\max \{n$, deg $b\}$ and since rank $\mathbb{P}_{n}^{\mathbf{f}}=d$ for all $n \geq d$, it follows from (2.6) that the rank of $\mathbb{P}_{n}^{s}$ stabilizes for large $n$. Therefore, $s$ is a finite Blaschke product so that the function $\widetilde{f}$ is rational and therefore it is analytic at $t_{0}$. Thus, $f$ and $f$ are two rational functions with the same Taylor coefficients at $t_{0}$. Therefore, $f \equiv \widetilde{f}$, which means that the problem $\mathbf{P}_{\infty}$ has only one solution in $\mathcal{G S}$. 
In the complementary subcase of (3) where $v\left(\mathbb{P}_{d}^{\mathbf{f}}\right)=\kappa$ and $\operatorname{det} \mathbb{P}_{d}^{\mathbf{f}} \neq 0$ for all $n \geq d$ (and, therefore, all structured matrices $\mathbb{P}_{n}^{\mathbf{x}}$ are positive definite), the problem (3.36) may be indeterminate or determinate depending on the convergence or divergence of certain positive series (see [11]). In the first case the problem $\mathbf{P}_{\infty}$ is indeterminate, since every $h \in \mathcal{S}$ subject to (3.36) leads via formula (3.34) to a solution $f$ to the problem $\mathbf{P}_{\infty}$ and since the transformation (3.34) is one-to-one. In the second case, it follows that the problem $\mathbf{P}_{\infty}$ has a unique solution $f \in \mathcal{G} \mathcal{S}$ with $\kappa$ poles inside $\mathbb{D}$; however, we do not know if it may or may not have solutions in $\mathcal{G S}$ with a larger pole multiplicity. A separate topic in interpolation theory for generalized Schur functions is to characterize all possible pole multiplicities for solutions of the problem and to find the minimally possible one. This issue will be addressed on a separate occasion.

\section{Appendix A. Asymptotics for eigenvalues of the matrix $\mathbb{P}_{n}^{b}$}

Theorem A.1. Let $b(z)=z^{m}$ and let $\lambda_{1} \leq \lambda_{2} \leq \cdots \leq \lambda_{n}$ be the eigenvalues of the matrix $\mathbb{P}_{n}^{b}$. Then

$$
\lambda_{r}=\frac{(n-r) !^{2}(2 r-1) !(2 r-2) !}{(r-1) !^{2}(n+r-1) !^{2}} m^{2 r-1}+O\left(m^{2 r-2}\right) .
$$

In particular, all eigenvalues tend to infinity as $m \rightarrow \infty$.

Proof. Recall that $\mathbb{P}_{n}^{b}$ is constructed via formulas (1.5)-(1.8) from the parameters

$$
b_{j}=\frac{b^{(j)}\left(t_{0}\right)}{j !}=\left(\begin{array}{c}
m \\
j
\end{array}\right) t_{0}^{m-j}
$$

Thus, $\mathbb{P}_{n}^{b}=\left[p_{i j}^{b}\right]_{i, j=1}^{n}=\mathbb{H}_{n}^{b} \Psi_{n}\left(t_{0}\right) \mathbb{U}_{n}^{\mathbf{b} *}$ and we can compute its entries using the formula (1.9) (with $b_{j}$ instead of $f_{j}$ ) and the explicit formula (1.7) for the numbers $\Psi_{\ell r}$ :

$$
\begin{aligned}
p_{i j}^{\mathbf{b}} & =\sum_{r=1}^{j}\left(\sum_{\ell=1}^{r} b_{i+\ell-1} \Psi_{\ell r}\right) \bar{b}_{j-r} \\
& =\sum_{r=1}^{j} \sum_{\ell=1}^{r}(-1)^{r-1}\left(\begin{array}{c}
m \\
i+\ell-1
\end{array}\right)\left(\begin{array}{c}
r-1 \\
\ell-1
\end{array}\right)\left(\begin{array}{c}
m \\
j-r
\end{array}\right) t_{0}^{j-i} \\
& =t_{0}^{j-i} \sum_{r=1}^{j}(-1)^{r-1}\left(\begin{array}{c}
m \\
i+r-1
\end{array}\right)\left(\begin{array}{c}
m-r \\
j-r
\end{array}\right) \\
& =t_{0}^{j-i} m^{i+j-1} \sum_{r=1}^{j} \frac{(-1)^{r-1}}{(i+r-1) !(j-r) !}+O\left(m^{i+j-2}\right) \\
& =\frac{t_{0}^{j-i} m^{i+j-1}}{(i-1) !(j-1) !(i+j-1)}+O\left(m^{i+j-2}\right) .
\end{aligned}
$$


For $1 \leq i_{1}<i_{2}<\cdots<i_{r} \leq n$, denote by $M_{i_{1} i_{2} \cdots i_{r}}$ the principal minor of the matrix $\mathbb{P}_{n}^{b}$ with rows and columns $i_{1}, \ldots, i_{r}$. Then

$$
\sum_{1 \leq i_{1}<i_{2}<\cdots<i_{r} \leq n} \lambda_{i_{1}} \lambda_{i_{2}} \cdots \lambda_{i_{r}}=\sum_{1 \leq i_{1}<i_{2}<\cdots<i_{r} \leq n} M_{i_{1} i_{2} \cdots i_{r}} .
$$

If $\left\{i_{1}, \ldots, i_{r}\right\} \neq\{n-r+1, \ldots, n\}$, then

$$
M_{i_{1} i_{2} \cdots i_{r}}=O\left(m^{\left(2 i_{1}-1\right)+\cdots+\left(2 i_{r}-1\right)}\right)=O\left(m^{r(2 n-r)-1}\right) .
$$

If $\left\{i_{1}, \ldots, i_{r}\right\}=\{n-r+1, \ldots, n\}$,

$$
\begin{aligned}
M_{i_{1} i_{2} \cdots i_{r}} & =\operatorname{det}\left[\frac{m^{i+j-1} t_{0}^{j-i}}{(i+j-1)(i-1) !(j-1) !}+O\left(m^{i+j-2}\right)\right]_{i, j=n-r+1}^{n} \\
& =\operatorname{det}\left[\frac{m^{i+j-1} t_{0}^{j-i}}{(i+j-1)(i-1) !(j-1) !}\right]_{i, j=n-r+1}^{n}+O\left(m^{r(2 n-r)-1}\right) \\
& =\operatorname{det}\left[m D \mathcal{H}_{n-r+1}^{n} D^{*}\right]+O\left(m^{r(2 n-r)-1}\right),
\end{aligned}
$$

where

$$
D=\operatorname{diag}\left(\frac{t_{0}^{-i+1} m^{i-1}}{(i-1) !}\right)_{i=n-r+1}^{n} \quad \text { and } \quad \mathcal{H}_{\ell}^{n}=\left[\frac{1}{i+j-1}\right]_{i, j=\ell}^{n} .
$$

The matrix $\mathcal{H}_{\ell}^{n}$ is a Hilbert-type matrix and it is known that

$$
\operatorname{det} \mathcal{H}_{\ell}^{n}=\frac{(n-\ell) ! !^{2}(n+\ell-2) ! !^{2}}{(2 n-1) ! !(2 \ell-3) ! !},
$$

where we use the notation $n ! !:=n !(n-1) ! \cdots 1$ !. Hence, if $\left\{i_{1}, \ldots, i_{r}\right\}=\{n-r+$ $1, \ldots, n\}$, we have from (A.4) that

$$
M_{i_{1} i_{2} \cdots i_{r}}=c_{n, r} m^{r(2 n-r)}+O\left(m^{r(2 n-r)-1}\right),
$$

where

$$
c_{n, r}=\frac{(n-r-1) ! !^{2}(r-1) ! !^{2}(2 n-r-1) ! !^{2}}{(n-1) ! !^{2}(2 n-1) ! !(2 n-2 r-1) ! !} .
$$

We now claim that, for $r=1, \ldots, n$,

$$
\lambda_{n-r+1}=\frac{c_{n, r}}{c_{n, r-1}} m^{2 n-2 r+1}+O\left(m^{2 n-2 r}\right) .
$$

We prove (A.7) by double induction, first on $n$, then on $r$. For all $n \geq 1$, if $r=1$, the claim is about the asymptotics of the largest eigenvalue of $\mathbb{P}_{n}^{b}$. From (A.1), the bottomright entry of $\mathbb{P}_{n}^{b}$ is $p_{n, n}^{\mathbf{b}}=\left(m^{2 n-1} /\left((n-1) !^{2}(2 n-1)\right)\right)+O\left(m^{2 n-2}\right)$, which dominates all other entries. Therefore, the largest eigenvalue of $\mathbb{P}_{n}^{b}$ is

$$
\lambda_{n}=\frac{1}{(n-1) !^{2}(2 n-1)} m^{2 n-1}+O\left(m^{2 n-2}\right)=\frac{c_{n, 1}}{c_{n, 0}} m^{2 n-1}+O\left(m^{2 n-2}\right) .
$$


Suppose $n \geq 2$ and we have proven the claim for all eigenvalues of $\mathbb{P}_{n-1}^{b}$. Denote the eigenvalues of $\mathbb{P}_{n-1}^{b}$ in increasing order by $\lambda_{0}^{\prime}, \lambda_{1}^{\prime}, \ldots, \lambda_{n-1}^{\prime}$, then we have the asymptotics $\lambda_{n-r+1}^{\prime}=\left(c_{n-1, r} / c_{n-1, r-1}\right) m^{2 n-2 r+1}+O\left(m^{2 n-2 r}\right)$. Assume also that the $r-1$ largest eigenvalues $(r \geq 1)$ of $\mathbb{P}_{n}^{b}$, namely $\lambda_{n}, \lambda_{n-1}, \ldots, \lambda_{n-r+2}$, all have asymptotics as described in (A.7). Note that $\mathbb{P}_{n-1}^{b}$ is the leading submatrix of $\mathbb{P}_{n}^{b}$. So by the Interlacing theorem,

$$
\lambda_{1} \leq \lambda_{1}^{\prime} \leq \lambda_{2} \leq \cdots \leq \lambda_{n-1}^{\prime} \leq \lambda_{n}
$$

Then, for $\left\{i_{1}, \ldots, i_{r}\right\} \neq\{n-r+1, \ldots, n\}$,

$$
\lambda_{i_{1}} \lambda_{i_{2}} \cdots \lambda_{i_{r}} \leq \lambda_{n} \cdots \lambda_{n-r+2} \lambda_{n-r} \leq \lambda_{n} \cdots \lambda_{n-r+2} \lambda_{n-r}^{\prime}=O\left(m^{r(2 n-r)-2}\right) .
$$

Substituting the estimates (A.8), (A.3) and (A.6) into the identity (A.2),

$$
\lambda_{n} \lambda_{n-1} \cdots \lambda_{n-r+1}+O\left(m^{r(2 n-r)-2}\right)=c_{r} m^{r(2 n-r)}+O\left(m^{r(2 n-r)-1}\right) .
$$

Dividing the above identity by the asymptotics for $\lambda_{n}, \ldots, \lambda_{n-r+2}$,

$$
\begin{aligned}
\lambda_{n-r+1} & =\frac{c_{n, r}}{c_{n, r-1}} m^{2 n-2 r+1}+O\left(m^{2 n-2 r}\right) \\
& =\frac{(r-1) !^{2}(2 n-2 r) !(2 n-2 r+1) !}{(n-r) !^{2}(2 n-r) !^{2}} m^{2 n-2 r+1}+O\left(m^{2 n-2 r}\right),
\end{aligned}
$$

which can be rewritten as

$$
\lambda_{r}=\frac{(n-r) !^{2}(2 r-1) !(2 r-2) !}{(r-1) !^{2}(n+r-1) !^{2}} m^{2 r-1}+O\left(m^{2 r-2}\right) .
$$

This completes the proof.

\section{References}

[1] J. A. Ball, 'Interpolation problems of Pick-Nevanlinna and Loewner type for meromorphic matrix functions', Integral Equations Operator Theory 6 (1983), 804-840.

[2] J. A. Ball, I. Gohberg and L. Rodman, Interpolation of Rational Matrix Functions (Birkhäuser Verlag, Basel, 1990).

[3] J. A. Ball and J. W. Helton, 'Interpolation problems of Pick-Nevanlinna and Loewner types for meromorphic matrix-functions: parametrization of the set of all solutions', Integral Equations Operator Theory 9 (1986), 155-203.

[4] V. Bolotnikov, 'A uniqueness result on boundary interpolation', Proc. Amer. Math. Soc. 136(5) (2008), 1705-1715.

[5] V. Bolotnikov, 'On higher order boundary derivatives of an analytic self-map of the unit disk', J. Approx. Theory 163(4) (2011), 568-589.

[6] V. Bolotnikov and H. Dym, 'On boundary interpolation for matrix Schur functions', Mem. Amer. Math. Soc. 181 (2006), 856.

[7] V. Bolotnikov and A. Kheifets, 'The higher order Carathéodory-Julia theorem and related boundary interpolation problems', Oper. Theory Adv. Appl. OT 179 (2007), 63-102.

[8] V. Bolotnikov and A. Kheifets, 'Carathéodory-Julia type conditions and symmetries of boundary asymptotics for analytic functions on the unit disk', Math. Nachr. 282(11) (2009), 1513-1536. 
[9] V. Bolotnikov, A. Kheifets and L. Rodman, 'Jet functions having indefinite Carathéodory-Pick matrices', Linear Algebra Appl. 385 (2004), 215-286.

[10] V. Bolotnikov and N. Zobin, 'Boundary asymptotic expansions of analytic self-maps of the unit disk', Rev. Mat. Iberoam., to appear.

[11] I. V. Kovalishina, 'A multiple boundary interpolation problem for contractive matrix-valued functions in the unit circle', Teor. Funktsii, Funktsional. Anal. i Ikh Prilozhen. 51 (1989), 38-55; Engl. transl. J. Soviet Math. 52(6) (1990), 3467-3481.

[12] M. G. Krĕn and H. Langer, 'Über die verallgemeinerten Resolventen und die charakteristische Funktion eines isometrischen Operators im Raume $\Pi_{\kappa}{ }^{\prime}$, Colloq. Math. Soc. János Bolyai 5 (1972), 353-399.

[13] M. G. Kreר̆n and H. Langer, 'Über einige Fortsetzungsprobleme, die eng mit der Theorie hermitescher Operatoren im Raume $\Pi_{\kappa}$ zusammenhängen. I. Einige Funktionenklassen und ihre Darstellungen', Math. Nachr. 77 (1977), 187-236.

[14] I. Schur, 'Über Potenzreihen, die im Innern des Einheitskreises beschränkt sind', J. reine angew. Math. 147 (1917), 205-232.

[15] T. Takagi, 'On an algebraic problem related to an analytic theorem of Carathéodory and Fejér and on an allied theorem of Landau', Jpn J. Math. 1 (1924), 83-93.

\author{
VLADIMIR BOLOTNIKOV, Department of Mathematics, \\ The College of William and Mary, Williamsburg, VA, USA \\ e-mail: vladi@math.wm.edu \\ TENGYAO WANG, Department of Mathematics, Princeton University, \\ Princeton, NJ, USA \\ e-mail: tengyaow@princeton.edu
}

JOSHUA M. WEISS, Department of Mathematics, Haverford College,

Haverford, PA, USA

e-mail: jweiss@haverford.edu 